\title{
On the regularity of stationary points of a nonlocal isoperimetric problem
}

\author{
DORIAN GOLDMAN \\ The New York Times Company, 620 Eighth Avenue, New York, NY, 10018, USA \\ E-mail:dorian.goldman@nytimes.com \\ ALEXANDER Volkmann \\ Rocket Internet SE, Johannisstraße 20, 10117 Berlin, Germany \\ E-mail: alexander.volkmann@rocket-internet.com
}

[Received 11 November 2014 and in revised form 15 August 2015]

\begin{abstract}
In this article we establish $C^{3, \alpha}$-regularity of the reduced boundary of stationary points of a nonlocal isoperimetric problem in a domain $\Omega \subset \mathbb{R}^{n}$. In particular, stationary points satisfy the corresponding Euler-Lagrange equation classically on the reduced boundary. Moreover, we show that the singular set has zero $(n-1)$-dimensional Hausdorff measure. This complements the results in [4] in which the Euler-Lagrange equation was derived under the assumption of $C^{2}$-regularity of the topological boundary and the results in [27] in which the authors assume local minimality. In case $\Omega$ has nonempty boundary, we show that stationary points meet the boundary of $\Omega$ orthogonally in a weak sense, unless they have positive distance to it.
\end{abstract}

2010 Mathematics Subject Classification: Primary 49Q10, 49Q20; Secondary 82B24.

Keywords: Ohta-Kawasaki energy, nonlocal isoperimetric problem, regularity theory, Caccioppoli sets, varifolds.

\section{Introduction}

The main goal of this work is to establish $C^{3, \alpha}$-regularity of the reduced boundary of stationary points of a nonlocal isoperimetric problem, and estimate the size of its singular set. More precisely, we consider the following functional

$$
\varepsilon_{\gamma}(E):=P(E, \Omega)+\gamma \int_{E} \int_{E} G(x, y) d y d x+\int_{E} f(x) d x
$$

where $\Omega \subset \mathbb{R}^{n}$ is a domain (open, connected) of class $C^{2}, E \subset \Omega$ is a bounded set of finite perimeter $P(E, \Omega)$ in $\Omega, \gamma \geqslant 0, f \in C_{l o c}^{2}(\bar{\Omega})$, and $G$ denotes a symmetric "kernel" (see below for precise assumptions on $G$ ). The reader should think of $G$ as the Green's function of the Laplace operator with Neumann boundary condition in $\Omega$ or the Newtonian potential in case $\Omega=\mathbb{R}^{n}$.

Physically, the first term in (1) models surface tension an thus its minimization favors clustering, whereas the second term can be used to model a competing repulsive term. The third term can be used to model additional external forces, cf. [12]. The functional $\varepsilon_{\gamma}$ is often referred to as the sharpinterface Ohta-Kawasaki energy [23] in connection with di-block copolymer melts. Minimizers of $\varepsilon_{\gamma}$ under a volume constraint describe a number of polymer systems [6, 22, 24] as well as many other physical systems $[3,7,13,18,22]$ due to the fundamental nature of the Coulombic term. Despite the abundance of physical systems for which (1) is applicable, rigorous mathematical 
analysis for the case $\gamma \neq 0$ is fairly recent. We refer to the introduction of [5] for more details and an account of the results about this functional.

Regularity for (local) minimizers of $\varepsilon_{\gamma}$ under a volume constraint was established by Sternberg and Topaloglu [27]. Sternberg and Topaloglu showed that any local minimizer $E$ of $\varepsilon_{\gamma}$ in a ball $B_{\rho}(x)$ is a so called $(K, \varepsilon)$-minimizer of perimeter in the sense that

$$
P\left(E, B_{\rho}(x)\right) \leqslant P\left(F, B_{\rho}(x)\right)+K \rho^{n-1+\varepsilon} \text { for all } F \text { such that } F \Delta E \subset \subset B_{\rho}(x),
$$

for some $K<\infty$ some $\varepsilon \in(0,1]$. Standard results (see for example [11, 19, 21]) imply that the reduced boundary $\partial^{*} E \cap B_{\rho}(x)$ is of class $C^{1, \frac{\varepsilon}{2}}$ and that the singular set $\left(\partial E \backslash \partial^{*} E\right) \cap B_{\rho}(x)$ has Hausdorff dimension at most $n-8$. Standard elliptic regularity theory then implies higher regularity.

For stationary points of $\varepsilon_{\gamma}$, which are not a priori minimizing in any sense, these methods are no longer available. To this end Röger and Tonegawa [25, Section 7.2] proved $C^{3, \alpha}$-regularity of the reduced boundary of stationary points of $\varepsilon_{\gamma}$ that arise as the limit of stationary points of the (diffuse) Ohta-Kawasaki energy with parameter $\varepsilon$ going to zero. They also showed that in this case the singular set has zero $(n-1)$-dimensional Hausdorff measure.

Our main result (Theorem 1.2) removes this special assumption. In particular, we do not require any minimality assumptions. As part of our proof we establish a weak measure theoretic form of the Euler-Lagrange equation for arbitrary stationary points of $\varepsilon_{\gamma}$ under very weak regularity assumptions (we only require the set to have finite perimeter). The Euler-Lagrange equation for stationary points of $\varepsilon_{\gamma}$ has previously been derived by Choksi and Sternberg [4], however assuming $C^{2}$-regularity of the topological boundary. An application of our main result is used in [14] which studies the asymptotics of stationary points of the Ohta-Kawasaki energy and its diffuse interface version.

In order to state our main result we need to introduce some notation and specify our hypotheses:

For a given domain $\Omega \subset \mathbb{R}^{n}$ with $C^{2}$-boundary we consider two classes of sets.

$$
Q:=\{E \subset \Omega: E \text { is bounded and } P(E, \Omega)<+\infty\} \quad \text { and } \quad Q_{m}:=\{E \in Q:|E|=m\},
$$

where $m \in(0,|\Omega|)$. A stationary point of $\varepsilon_{\gamma}$ in $Q$ or $Q_{m}$ is then defined as follows.

DEFINITION 1.1 (stationary point of $\varepsilon_{\gamma}$ ) A set $E \in Q$ is said to be a stationary point of $\varepsilon_{\gamma}$ (see (1)) in $Q$ if for every vector field $X \in C_{c}^{1}\left(\mathbb{R}^{n} ; \mathbb{R}^{n}\right)$ with $X \cdot v_{\Omega}=0$ on $\partial \Omega$ we have that

$$
\left.\frac{d}{d t}\right|_{t=0} \varepsilon_{\gamma}\left(\phi_{t}(E)\right)=0
$$

where $\left\{\phi_{t}\right\}$ is the flow of $X$, i.e. $\partial_{t} \phi_{t}=X \circ \phi_{t}, \phi_{0}=$ id. If (2) holds only for all $X$ such that $\phi_{t}(E) \in Q_{m}$ for all $t \in(-\varepsilon, \varepsilon)$ and some small $\varepsilon>0$, then we call $E$ a stationary point of $\varepsilon_{\gamma}$ in $Q_{m}$.

We now specify the assumptions that we impose on the function $G$ appearing in (1).

Firstly, we let $\Gamma$ be the fundamental solution of the Laplace operator given by

$$
\Gamma(x, y):= \begin{cases}\frac{1}{\omega_{n}(n-2)} \frac{1}{|x-y|^{n-2}}, & n \geqslant 3 \\ -\frac{1}{2 \pi} \log |x-y|, & n=2 .\end{cases}
$$


Here $\omega_{n}=\mathcal{H}^{n}\left(\mathbb{S}^{n}\right)$. We assume that

$$
G(x, y)=\Gamma(x, y)+R(x, y)
$$

where $R$ is a symmetric corrector function. I.e.

$$
\begin{cases}\Delta R(\cdot, y)=\frac{1}{\mid \Omega} & \text { in } \Omega \\ \frac{\partial R(\cdot, y)}{\partial \nu_{\Omega}}=-\frac{\partial \Gamma(\cdot, y)}{\partial \nu_{\Omega}} & \text { on } \partial \Omega\end{cases}
$$

for all $y \in \Omega$. Here we interpret $|\Omega|^{-1}$ to be zero for domains $\Omega$ of infinite measure. In case $\Omega$ is bounded $G$ is a Neumann Green's function of the Laplace operator. In case $\Omega=\mathbb{R}^{2}$ we also allow that $G(x, y)=\Gamma_{\beta}(x, y)$ for $\beta \in(0,1)$, where $\Gamma_{\beta}(x, y):=|x-y|^{-\beta}$.

For a bounded Borel set $E \subset \Omega$ we define

$$
\phi_{E}(x):=\int_{E} G(x, y) d y
$$

to be the potential of $E$ associated to the kernel $G$. By standard elliptic theory we have $\phi_{E} \in$ $C_{l o c}^{1, \alpha}(\bar{\Omega})$.

Our main result reads as follows.

THEOREM 1.2 Let $E$ be a stationary point of the functional $\varepsilon_{\gamma}$ in $Q$ or $Q_{m}$ with $f$ and $G$ as above. Then the reduced boundary $\partial_{\Omega}^{*} E=\partial^{*} E \cap \Omega$ is of class $C^{3, \alpha}$ for all $\alpha \in(0,1)$. In particular, the equation

$$
H+2 \gamma \phi_{E}+f=\lambda
$$

holds classically on $\partial_{\Omega}^{*} E$ where $H$ is the mean curvature ${ }^{1}$ of $\partial_{\Omega}^{*} E, \lambda$ is a Lagrange multiplier, and $\phi_{E}$ is the potential arising from $E$, given by (3). (When $E$ is a stationary point in the class $Q$, then $\lambda=0$.) The measure $\mu_{E}=\mathcal{H}^{n-1}\left\llcorner\partial_{\Omega}^{*} E\right.$ is weakly orthogonal to $\partial \Omega$ in the sense that

$$
\int_{\partial_{\Omega}^{*} E} \operatorname{div}_{E} X d \mathcal{H}^{n-1}=-\int_{\partial_{\Omega}^{*} E} \vec{H} \cdot X d \mathcal{H}^{n-1},
$$

for all $X \in C_{c}^{1}\left(\mathbb{R}^{n} ; \mathbb{R}^{n}\right)$ with $X \cdot v_{\Omega}=0$ on $\partial \Omega$.

Moreover, the singular set $\left(\partial E \backslash \partial^{*} E\right) \cap \Omega$ is a relatively closed subset of $\partial E \cap \Omega$ which satisfies $\mathcal{H}^{s}\left(\left(\partial E \backslash \partial^{*} E\right) \cap \Omega\right)=0$ for all $s \geqslant n-1$.

REMARK 1.3 The estimate on the singular set in Theorem 1.2 cannot be improved to $s \geqslant n-2$. This can already be seen in the case $\gamma=0$. E.g. let $\Omega=B_{1}(0) \subset \mathbb{R}^{n}$ and set $E:=\{x=$ $\left.\left(x_{1}, \ldots, x_{n}\right) \in \Omega: x_{1} \cdot x_{2}>0\right\}$. Then $E$ is a stationary point of the perimeter functional with singular set $\left(\partial E \backslash \partial^{*} E\right) \cap \Omega=\left\{x \in \Omega: x_{1}=x_{2}=0\right\}$.

Our paper is organized as follows. In Section 2 we introduce our notation and review the basic theory of rectifiable varifolds and sets of finite perimeter, and present Allard's regularity theorem and De

\footnotetext{
1 By our convention the mean curvature is chosen such that the boundary of the unit ball in $\mathbb{R}^{n}$ has positive mean curvature equal to $n-1$.
} 
Giorgi's structure theorem for the reader's convenience. In Section 3 we prove some preliminary results that are needed in order to prove Theorem 1.2. In Section 4 we prove Theorem 1.2. In Section 5 we include, for convenience, the regularity for local minimizers of $\varepsilon_{\gamma}$ near boundary points $x \in \partial E \cap \partial \Omega$. This has already been proven independently by Julin and Pisante [17, Theorem 3.2].

\section{Notation and preliminaries}

Throughout this work we assume that $\Omega \subset \mathbb{R}^{n}, n \geqslant 2$, is a domain (open, connected) of class $C^{2}$ (although the regularity assumption on the boundary is only needed when we consider vector fields that do not have compact support inside $\Omega$ ). In this section we introduce our notation and summarize basic results from geometric measure theory that are needed in the sequel. For more details on the subject we refer the reader to $[8,11,19,26]$.

\subsection{Varifolds and Allard's regularity theorem}

Here we collect basic definitions for varifolds and state Allard's regularity theorem. An $\mathcal{H}^{k}$ measurable set $M \subset \mathbb{R}^{n}$ is called countably $k$-rectifiable if

$$
M \subset \bigcup_{j=0}^{\infty} N_{j}
$$

where $N_{j} \subset \mathbb{R}^{n}, 0 \leqslant j \leqslant n-1$, are $k$-dimensional submanifolds of class $C^{1}$ and $\mathcal{H}^{k}\left(N_{0}\right)=0$. For a vector field $X \in C_{c}^{1}\left(\mathbb{R}^{n} ; \mathbb{R}^{n}\right)$ we can define the tangential divergence $\operatorname{div}_{M} X$ of $X$ by setting

$$
\operatorname{div}_{M} X(x):=\operatorname{div}_{N_{j}} X(x)
$$

for $x \in N_{j}$, which is well-defined $\mathcal{H}^{k}$-a.e. on $M$. Here $\operatorname{div}_{N_{j}} X(x)=\sum_{i=1}^{k} \tau_{i} \cdot D X(x) \tau_{i}$, where $\left\{\tau_{i}\right\}_{i=1, \ldots, k}$ is an orthonormal basis of the tangent plane $T_{x} N_{j}$ of $N_{j}$ at the point $x$.

For the purpose of this article we use the following pragmatic definition of rectifiable $k$ varifolds, which usually has to be deduced from the definition (we refer to [26] for details):

A rectifiable $k$-varifold $\mu$ in $\Omega$ is a Radon measure on $\Omega$ such that

$$
\mu=\theta \mathcal{H}_{\llcorner}^{k} M,
$$

where $M$ is a countably $k$-rectifiable set and where the multiplicity function $\theta \in L_{\text {loc }}^{1}\left(\mathcal{H}^{k}\llcorner M)\right.$ is such that $\theta>0 \mathcal{H}^{k}$-a.e. on $M$.

The first variation $\delta \mu$ of $\mu$ with respect to $X \in C_{c}^{1}\left(\Omega, \mathbb{R}^{n}\right)$ is given by

$$
\delta \mu(X):=\int_{M} \operatorname{div}_{M} X d \mu,
$$

which by $[26, \S 16]$ is equal to $\left.\frac{d}{d t}\left(\phi_{t \sharp} \mu\right)(\Omega)\right|_{t=0}$. Here $\phi_{t \sharp} \mu$ denotes the image varifold given by $\phi_{t \sharp} \mu:=\left(\theta \circ \phi_{t}^{-1}\right) \mathcal{H}^{k}\left\llcorner\phi_{t}(M)\right.$, and where $\left\{\phi_{t}\right\}$ denotes the flow of $X$.

We say that $\mu$ has generalized mean curvature $\vec{H}$ in $\Omega$ if

$$
\delta \mu(X)=\int_{M} \operatorname{div}_{M} X d \mu=-\int_{M} \vec{H} \cdot X d \mu \quad \text { for all } X \in C_{c}^{1}\left(\Omega ; \mathbb{R}^{n}\right),
$$


where $\vec{H}$ is a locally $\mu$-integrable function on $M \cap \Omega$ with values in $\mathbb{R}^{n}$. We remark that using the Riesz representation theorem such an $\vec{H}$ exists if the total variation $\|\delta \mu\|$ is a Radon measure in $\Omega$ and moreover $\|\delta \mu\|$ is absolutely continuous with respect to $\mu$ (see [26] for details).

We make the trivial but important remark that a rectifiable $k$-varifold $\mu$ in $\Omega$ that has finite total mass $\mu(\Omega)$ naturally defines a rectifiable $k$-varifold in $\mathbb{R}^{n}$.

A fundamental result in the theory of varifolds is the following regularity theorem due to Allard [1] (see also [26, Chapter 5] for a more accessible approach) that holds for rectifiable $k$-varifolds $\mu$ in $\Omega \subset \mathbb{R}^{n}$. We use the following hypotheses.

$$
\left.\begin{array}{c}
1 \leqslant \theta \quad \mu \text {-a.e. }, 0 \in \operatorname{spt}(\mu), B_{\rho}(0) \subset \Omega \\
\alpha_{k}^{-1} \rho^{-k} \mu\left(B_{\rho}(0)\right) \leqslant 1+\delta \\
\left(\int_{B_{\rho}(0)}|\vec{H}|^{p} d \mu\right)^{\frac{1}{p}} \rho^{1-\frac{k}{p}} \leqslant \delta .
\end{array}\right\}
$$

Here $\alpha_{k}$ denotes the $k$-dimensional volume of the unit ball in $\mathbb{R}^{k}$. Note that $\alpha_{k}=\frac{\omega_{k}}{k}$.

THEOREM 2.1 (Allard's Regularity Theorem) For $p>k$, there exist $\delta=\delta(n, k, p)$ and $\gamma=$ $\gamma(n, k, p) \in(0,1)$ such that if $\mu$ is a rectifiable $k$-varifold in $\Omega$ that has generalized mean curvature $\vec{H}$ in $\Omega$ (see (5)) and satisfies hypotheses (h), then $\operatorname{spt}(\mu) \cap B_{\gamma \rho}(0)$ is a graph of a $C^{1,1-\frac{k}{p}}$ function with scaling invariant $C^{1,1-\frac{k}{p}}$ estimates depending only on $n, k, p, \delta$.

REMARK 2.2 More precisely, there is a linear isometry $q$ of $\mathbb{R}^{n}$ and a function $u \in$ $C^{1,1-\frac{k}{p}}\left(B_{\gamma r}^{k}(0) ; \mathbb{R}^{n-k}\right)$ with $u(0)=0, \operatorname{spt}(\mu) \cap B_{\gamma \rho}(0)=q(\operatorname{graph}(u)) \cap B_{\gamma \rho}(0)$, and

$\rho^{-1} \sup _{B_{\gamma \rho}^{k}(0)}|u|+\sup _{B_{\gamma \rho}^{k}(0)}|D u|+\rho^{1-\frac{k}{p}} \sup _{\substack{x, y \in B_{\gamma \rho}^{k}(0) \\ x \neq y}}|x-y|^{-\left(1-\frac{k}{p}\right)}|D u(x)-D u(y)| \leqslant c(n, k, p) \delta^{1 / 4 k}$.

\subsection{Sets of finite perimeter}

Let $E \subset \Omega$ be a Borel set. We say that $E$ has finite perimeter $P(E, \Omega)$ in $\Omega$ if

$$
P(E, \Omega):=\sup _{\substack{X \in C_{c}^{1}\left(\Omega ; \mathbb{R}^{n}\right) \\|X| \leqslant 1}} \int_{E} \operatorname{div} X d x<\infty .
$$

The Riesz representation theorem implies the existence of a Radon measure $\mu_{E}$ on $\Omega$ and a $\mu_{E}$ measurable vector field $\eta_{E}: \Omega \rightarrow \mathbb{R}^{n}$ with $\left|\eta_{E}\right|=1 \mu_{E}$-a.e. such that

$$
\int_{E} \operatorname{div} X d x=\int_{\mathbb{R}^{n}} X \cdot \eta_{E} d \mu_{E} \quad \text { for all } X \in C_{c}^{1}\left(\Omega ; \mathbb{R}^{n}\right) .
$$

The vector valued measure $\vec{\mu}_{E}:=\eta_{E} \mu_{E}$ is sometimes referred to as the Gauss-Green measure of $E$ (with respect to $\Omega$ ). For the total perimeter of the set $E$ in $\Omega$ we have

$$
P(E, \Omega)=\mu_{E}(\Omega) .
$$


In the case that $\partial E \cap \Omega$ is of class $C^{1}$, we have

$$
\vec{\mu}_{E}=v_{E} \mathcal{H}^{n-1}\left\llcorner(\partial E \cap \Omega) \text { and } P(E, \Omega)=\mathcal{H}^{n-1}(\partial E \cap \Omega) .\right.
$$

In particular, we have for every point $x \in \partial E \cap \Omega$

$$
v_{E}(x)=\lim _{r \rightarrow 0} f_{\partial E \cap B_{r}(x)} v_{E} d \mathcal{H}^{n-1}=\lim _{r \rightarrow 0} \frac{\vec{\mu}_{E}\left(B_{r}(x)\right)}{\mu_{E}\left(B_{r}(x)\right)} .
$$

For a generic set $E$ of finite perimeter, the reduced boundary $\partial_{\Omega}^{*} E$ of $E$ in $\Omega$ is defined as those $x \in \partial E \cap \Omega$ such that the above limit on the right hand side exists and has norm 1 . The Lebesgue-Besicovitch differentiation theorem implies that $\mu_{E}\left(\mathbb{R}^{n} \backslash \partial_{\Omega}^{*} E\right)=0$. The vector field $v_{E} \in L^{1}\left(\mu_{E} ; \mathbb{R}^{n}\right)$ defined by the equation (7) on $\partial_{\Omega}^{*} E$ (and set to 0 elsewhere), is called the measure theoretic outer unit normal of $E$. For more details on sets of finite perimeter we refer to [8, 11, 26].

THEOREM 2.3 (De Giorgi's structure theorem) Suppose $E$ has finite perimeter in $\Omega$. Then $\partial_{\Omega}^{*} E$ is countably $(n-1)$-rectifiable. In addition for all $x \in \partial_{\Omega}^{*} E$

$$
\Theta\left(\mu_{E}, x\right):=\lim _{r \rightarrow 0} \frac{\mu_{E}\left(B_{r}(x)\right)}{\alpha_{n-1} r^{n-1}}=1,
$$

where $\alpha_{n-1}$ is the volume of the unit ball in $\mathbb{R}^{n-1}$. (i.e. the limit exists and is equal to 1.) Moreover, $\mu_{E}=\mathcal{H}^{n-1}\left\llcorner\partial_{\Omega}^{*} E\right.$.

REMARK 2.4 De Giorgi's structure theorem in particular shows that every set $E$ of finite perimeter defines - through its generalized surface measure $\mu_{E}$ - a rectifiable $(n-1)$-varifold of multiplicity $\theta \equiv 1$ on $\partial_{\Omega}^{*} E$.

Let $E \subset \Omega$ be of finite perimeter in $\Omega$. If $\Omega$ is Lipschitz regular, one can define the (inner) trace $\chi_{E}^{+} \in L^{1}\left(\mathcal{H}^{n-1}\llcorner\partial \Omega)\right.$ of $\chi_{E}$ on $\partial \Omega$. For details we refer to [8, Chapter 5.3]. For every vector field $X \in C_{c}^{1}\left(\mathbb{R}^{n} ; \mathbb{R}^{n}\right)$ we have

$$
\int_{E} \operatorname{div} X d x=\int_{\partial_{\Omega}^{*} E} X \cdot v_{E} d \mathcal{H}^{n-1}+\int_{\partial \Omega} X \cdot v_{\Omega} \chi_{E}^{+} d \mathcal{H}^{n-1} .
$$

This implies that $E$ is also a set of finite perimeter as a subset of $\mathbb{R}^{n}$ with

$$
P\left(E, \mathbb{R}^{n}\right)=P(E, \Omega)+\int_{\partial \Omega}\left|\chi_{E}^{+}\right| d \mathcal{H}^{n-1} .
$$

As a finite perimeter set in $\mathbb{R}^{n}, E$ also has a Gauss-Green measure which we shall denote by $\vec{\mu}_{E}^{*}$. Obviously $\vec{\mu}_{E}^{*}\left\llcorner\Omega=\vec{\mu}_{E}\right.$ and $\partial^{*} E \cap \Omega=\partial_{\Omega}^{*} E$.

Since sets of finite perimeter are equivalence classes of sets, one needs to choose a good representative in order to talk about their regularity properties. Without loss of generality (see [11, Proposition 3.1] for details) we will always assume that any finite perimeter set $E$ at hand satisfies the following properties:

$E$ is Borel

$$
\begin{aligned}
& 0<\left|E \cap B_{\rho}(x)\right|<\left|B_{\rho}(x)\right| \text { for all } x \in \partial E \text { and all } \rho>0 \\
& \overline{\partial_{\Omega}^{*} E}=\overline{\partial E \cap \Omega} \text { which implies that } \operatorname{spt}\left(\mu_{E}\right)=\overline{\partial E \cap \Omega} .
\end{aligned}
$$




\subsection{The first variation of perimeter}

Let $X \in C_{c}^{1}\left(\Omega ; \mathbb{R}^{n}\right)$ with corresponding flow $\left\{\phi_{t}\right\}$. The first variation of perimeter is then easily computed as (see $[11,19])$

$$
\left.\frac{d}{d t}\right|_{t=0} P\left(\phi_{t}(E), \Omega\right)=\int_{\partial_{\Omega}^{*} E} \operatorname{div}_{E} X d \mathcal{H}^{n-1},
$$

where $\operatorname{div}_{E} X$ is the tangential divergence of the vector field $X$ with respect to $E$ :

$$
\operatorname{div}_{E} X=\operatorname{div} X-v_{E} \cdot D X v_{E},
$$

which obviously agrees with the definition of tangential divergence with respect to $\partial_{\Omega}^{*} E$. Hence, the expression (11) equals the first variation $\delta \mu_{E}(X)$ of the varifold $\mu_{E}$ with respect to $X$.

In order to investigate the behavior of stationary points $E$ of $\varepsilon_{\gamma}$ at the boundary $\partial \Omega$ of $\Omega$ we need to allow for more general variations (as already appearing in Definition 1.1). By the regularity assumption on $\partial \Omega$ it follows (cf. [16]) that $\phi_{t}(\Omega) \equiv \Omega$ (and $\phi_{t}(\partial \Omega) \equiv \partial \Omega$ ) for the flow $\left\{\phi_{t}\right\}$ of any vector field $X \in C_{c}^{1}\left(\mathbb{R}^{n} ; \mathbb{R}^{n}\right)$ such that $X \cdot v_{\Omega}=0$ on $\partial \Omega$. Since $P\left(\phi_{t}(E), \Omega\right) \equiv\left(\phi_{t \sharp} \mu_{E}\right)\left(\mathbb{R}^{n}\right)$ we see that the formula (11) still holds for such vector fields $X$.

\section{Preliminary results}

Proposition 3.1 (First variation of nonlocal perimeter) Let $E \in Q$ and let $X \in C_{c}^{1}\left(\mathbb{R}^{n} ; \mathbb{R}^{n}\right)$ with $X \cdot v_{\Omega}=0$ on $\partial \Omega$ be a vector field with corresponding flow $\left\{\phi_{t}\right\}$. Then

$$
\begin{aligned}
\left.\frac{d}{d t} \varepsilon_{\gamma}\left(\phi_{t}(E)\right)\right|_{t=0}=\int_{\partial_{\Omega}^{*} E} \operatorname{div}_{E} X d \mathcal{H}^{n-1}+2 \gamma \int_{\partial_{\Omega}^{*} E} \phi_{E} X \cdot v_{E} d \mathcal{H}^{n-1} & \\
& +\int_{\partial_{\Omega}^{*} E} f X \cdot v_{E} d \mathcal{H}^{n-1} .
\end{aligned}
$$

Proof. The first variation of perimeter is equation (11). It remains to compute the first variation of the nonlocal term; the computation of the first variation of the third term is similar but easier. By the change of variables formula it holds that

$$
\int_{\phi_{t}(E)} \int_{\phi_{t}(E)} G(x, y) d x d y=\int_{E} \int_{E} G\left(\phi_{t}(x), \phi_{t}(y)\right)\left|\operatorname{det} D \phi_{t}(x)\right|\left|\operatorname{det} D \phi_{t}(y)\right| d x d y .
$$

Hence, we compute using (12) and the assumptions on $G$ which allow us to differentiate under the integral

$$
\begin{aligned}
\left.\frac{d}{d t}\right|_{t=0} & \int_{\phi_{t}(E)} \int_{\phi_{t}(E)} G(x, y) d x d y \\
& =2 \int_{E} \int_{E}\left(\nabla_{x} G\right)(x, y) \cdot X(x) d x d y+2 \int_{E} \int_{E} G(x, y) \operatorname{div} X(x) d x d y \\
& =2 \int_{E} \int_{E} \operatorname{div}(G(\cdot, y) X)(x) d x d y \\
& =2 \int_{E} \int_{E} \operatorname{div}(\Gamma(\cdot, y) X)(x) d x d y+2 \int_{E} \int_{\partial_{\Omega}^{*} E} R(\cdot, y) X \cdot v_{E} d \mu_{E} d y
\end{aligned}
$$


where in the last line we used the trace formula (9) and the fact that $v_{\Omega} \cdot X=0$ on $\partial \Omega$. We cannot directly apply the divergence theorem to the first term of (13) since $\partial_{\Omega}^{*} E$ is only $(n-1)$-rectifiable and $G(\cdot, y)$ is not of class $C^{1}$ near $x=y$. We can get around this technical obstacle by applying the results of [2], but we present a simple argument which suffices in our case. Since $\Omega$ is of class $C^{2}$ (for this argument Lipschitz is enough) we have (see Section 3) that $E$ is a set of finite perimeter in $\mathbb{R}^{n}$. By [11, Theorem 1.24] we can approximate $E$ in the support of $X$ by smooth sets $E^{i}$ such that

$$
\begin{aligned}
& \chi_{E^{i}} \rightarrow \chi_{E} \text { in } L_{l o c}^{1}\left(\mathbb{R}^{n}\right) \text { and } \\
& \vec{\mu}_{E^{i}}^{*} \rightarrow \vec{\mu}_{E}^{*} \text { weakly as Radon measures on } \mathbb{R}^{n} .
\end{aligned}
$$

We may apply the Lebesgue dominated convergence theorem to conclude that

$$
\int_{E} \int_{E} \operatorname{div}(\Gamma(\cdot, y) X)(x) d x d y=\lim _{i \rightarrow \infty} \int_{E} \int_{E^{i}} \operatorname{div}(\Gamma(\cdot, y) X)(x) d x d y .
$$

Moreover, we have

$$
\int_{E^{i}} \operatorname{div}(\Gamma(\cdot, y) X)(x) d x=\lim _{\rho \rightarrow 0} \int_{E^{i} \backslash B_{\rho}(y)} \operatorname{div}(\Gamma(\cdot, y) X)(x) d x .
$$

We may now apply the divergence theorem and we have for a.e. $0<\rho<1$

$$
\begin{aligned}
\int_{E^{i} \backslash B_{\rho}(y)} & \operatorname{div}(\Gamma(\cdot, y) X)(x) d x \\
& =\int_{\partial E^{i} \backslash B_{\rho}(y)} \Gamma(\cdot, y) X \cdot v_{E^{i}} d \mathcal{H}^{n-1}-\int_{\partial B_{\rho}(y) \cap E^{i}} \Gamma(\cdot, y) X \cdot v_{B_{\rho}(y)} d \mathcal{H}^{n-1} .
\end{aligned}
$$

The second term on the right hand side of (16) can be estimated by $c(n) \sup |X| \rho^{1-\varepsilon}$ for some $\varepsilon \in[0,1)$, and hence goes to zero as $\rho \rightarrow 0$. On the other hand, we have

$$
\begin{aligned}
& \mid \int_{\partial E^{i} \backslash B_{\varrho}(y)} \Gamma(\cdot, y) X \cdot v_{E^{i}} d \mathcal{H}^{n-1}-\int_{\partial E^{i}} \Gamma(\cdot, y) X \cdot v_{E^{i}} d \mathcal{H}^{n-1} \mid \\
& \leqslant \mathcal{H}^{n-1}\left(\partial E^{i} \cap B_{\varrho}(y)\right)^{1-\frac{1}{p}}\left(\int_{\partial E^{i} \cap \operatorname{spt}(X)}|\Gamma(\cdot, y)|^{p} d \mathcal{H}^{n-1}\right)^{\frac{1}{p}} \sup |X|,
\end{aligned}
$$

where $p \in\left(1, \frac{n-1}{n-2}\right)$, in case $n \geqslant 3$, and $p \in\left(1, \beta^{-1}\right)$ in case $G=\Gamma_{\beta}$. Whence, upon combining (15) and (16),

$$
\int_{E^{i}} \operatorname{div}(\Gamma(\cdot, y) X)(x) d x=\int_{\partial E^{i}} \Gamma(\cdot, y) X \cdot v_{E_{i}} d \mathcal{H}^{n-1} .
$$

Using (13) and applying Fubini's theorem we arrive at

$$
\int_{E} \int_{E^{i}} \operatorname{div}(G(\cdot, y) X)(x) d x d y=\int_{\partial E^{i}} \phi_{E} X \cdot v_{E^{i}} d \mathcal{H}^{n-1} .
$$

Now let $i \rightarrow \infty$, using the fact that $\phi_{E}$ is continuous and that $X$ has compact support, and combining (14) and (17) we obtain

$$
\int_{E} \int_{E} \operatorname{div}(G(\cdot, y) X)(x) d x d y=\int_{\partial^{*} E} \phi_{E} X \cdot v_{E} d \mathcal{H}^{n-1} .
$$

The claim now follows from the fact that $X$ is tangential to $\partial \Omega$. 
LEMma 3.2 There exists a vector field $Y \in C_{c}^{1}\left(\Omega ; \mathbb{R}^{n}\right)$ such that $\int_{E} \operatorname{div} Y d x=1$.

Proof. Assume by contradiction that for every vector field $X \in C_{c}^{1}\left(\Omega ; \mathbb{R}^{n}\right): \int_{E} \operatorname{div} X d x=0$. Then by Du Bois-Reymond's lemma [9] we conclude that

$$
\chi_{E}=0 \quad \text { or } \chi_{E}=1 \quad \aleph^{n} \text {-a.e. on } \Omega,
$$

where we used that $\Omega$ is connected. Hence,

$$
E=\Omega \text { or } E=\emptyset \text { in the measure theoretic sense. }
$$

This contradicts the assumption that $0<|E|<|\Omega|$, proving the claim.

PROPOSITION 3.3 (Euler-Lagrange equation of non-local perimeter) Let $E$ be a stationary point of $\varepsilon_{\gamma}$ in $Q_{m}$ or $Q$. Then there exists a real number $\lambda$ such that $\mu_{E}$ has a generalized mean curvature vector

$$
\vec{H}=-\left(\lambda-2 \gamma \phi_{E}-f\right) v_{E}
$$

and such that $\mu_{E}$ is weakly orthogonal to $\partial \Omega$. That is, for every vector field $X \in C_{c}^{1}\left(\mathbb{R}^{n} ; \mathbb{R}^{n}\right)$ with $X \cdot v_{\Omega}=0$ on $\partial \Omega$ the following variational equation is true:

$$
\int_{\partial_{\Omega}^{*} E} \operatorname{div}_{E} X d \mathcal{H}^{n-1}=-\int_{\partial_{\Omega}^{*} E} \vec{H} \cdot X d \mathcal{H}^{n-1} .
$$

For stationary points in $Q$, we have $\lambda=0$.

Proof.

Step 1: Construction of the local variation. The case of variations in $Q$ is an immediate consequence of Proposition 3.1. For the case of $Q_{m}$ let $Y \in C_{c}^{1}\left(\Omega ; \mathbb{R}^{n}\right)$ be a vector field such that $\int_{E} \operatorname{div} Y d x=$ 1. The existence of such a vector field is guaranteed by Lemma 3.2. Let $\left\{\phi_{t}\right\}$ be the flow of $X$ and $\left\{\psi_{s}\right\}$ the flow of $Y$. For $(t, s) \in \mathbb{R}^{2}$ set

$$
A(t, s):=P\left(\psi_{s}\left(\phi_{t}(E)\right), \Omega\right)
$$

and

$$
\mho(t, s):=\left|\psi_{s}\left(\phi_{t}(E)\right)\right|-|E| .
$$

Then $v \in C^{1}\left(\mathbb{R}^{2}\right), v(0,0)=0$ and $\partial_{s} v(0,0)=\int_{E} \operatorname{div} Y d x=1$. The implicit function theorem ensures the existence of an open interval $I$ containing 0 and a function $\sigma \in C^{1}(I)$ such that

$$
\mho(t, \sigma(t))=0 \text { for all } t \in I \text { and } \sigma^{\prime}(0)=-\frac{\partial_{t} v(0,0)}{\partial_{s} v(0,0)} .
$$

Hence,

$$
t \mapsto \psi_{\sigma(t)} \circ \phi_{t}
$$

is a 1-parameter family of $C^{1}$-diffeomorphisms of $\bar{\Omega}$ and thus defines a volume preserving variation of $E$ in $\Omega$. 
Step 2: Computing the first variation. The fact that $E$ is a stationary point in the class $Q_{m}$ then implies from Proposition 3.1

$$
\left.\frac{d}{d t}\right|_{t=0} A(t, \sigma(t))=\int_{\partial_{\Omega}^{*} E} \operatorname{div}_{E} X=-2 \gamma \int_{\partial_{\Omega}^{*} E} \phi_{E} X \cdot v_{E} d \mathcal{H}^{n-1}-\int_{\partial_{\Omega}^{*} E} f X \cdot v_{E} d \mathcal{H}^{n-1} .
$$

On the other hand, we have

$$
\begin{aligned}
\left.\frac{d}{d t}\right|_{t=0} A(t, \sigma(t)) & =\partial_{t} Q(0,0)+\sigma^{\prime}(0) \partial_{s} Q(0,0) \\
& =\int_{\partial_{\Omega}^{*} E} \operatorname{div}_{E} X d \mathcal{H}^{n-1}+\sigma^{\prime}(0) \int_{\partial_{\Omega}^{*} E} \operatorname{div}_{E} Y d \mathcal{H}^{n-1} \\
& =\int_{\partial_{\Omega}^{*} E} \operatorname{div}_{E} X d \mathcal{H}^{n-1}-\frac{\int_{E} \operatorname{div} X d x}{\int_{E} \operatorname{div} Y d x} \int_{\partial_{\Omega}^{*} E} \operatorname{div}_{E} Y d \mathcal{H}^{n-1} \\
& =\int_{\partial_{\Omega}^{*} E} \operatorname{div}_{E} X d \mathcal{H}^{n-1}-\lambda \int_{\partial_{\Omega}^{*} E} X \cdot v_{E} d \mathcal{H}^{n-1}
\end{aligned}
$$

where $\lambda:=\int_{\partial_{\Omega}^{*} E} \operatorname{div}_{E} Y d \mathcal{H}^{n-1}$, and where we used the divergence theorem on the last line. Therefore, setting $\vec{H}:=\left(2 \gamma \phi_{E}+f-\lambda\right) v_{E}$, we have

$$
\int_{\partial_{\Omega}^{*} E} \operatorname{div}_{E} X d \mathcal{H}^{n-1}=-\int_{\partial_{\Omega}^{*} E} \vec{H} \cdot X d \mathcal{H}^{n-1}
$$

for every vector field $X \in C_{c}^{1}\left(\mathbb{R}^{n} ; \mathbb{R}^{n}\right)$ with $X \cdot v_{\Omega}=0$ on $\partial \Omega$.

\section{Proof of the Theorem 1.2}

Firstly, notice that the weak orthogonality of $\mu_{E}$ and $\Omega$ is included in Proposition 3.3.

We want to apply Allard's regularity theorem (here Theorem 2.1) to establish the regularity of the reduced boundary $\partial_{\Omega}^{*} E$. We verify the necessary hypotheses:

By De Giorgi's structure theorem (here Theorem 2.3) and Remark 2.4 we have that $\mu_{E}$ is a multiplicity-1 rectifiable $(n-1)$-varifold. Moreover, for each point $x \in \partial_{\Omega}^{*} E$ we have that $\Theta\left(\mu_{E}, x\right)=1$. Now, we choose any point $x_{0} \in \partial_{\Omega}^{*} E$. Without loss of generality, after possibly translating and rotating the set $E$, we may assume that $x_{0}=0$ and $v_{E}(0)=-e_{n}$. We fix any $p>n-1$ and pick $\delta \in(0,1)$ to be as in the statement of Theorem 2.1. Since $\Theta\left(\mu_{E}, 0\right)=1$ we can find a small radius $\rho>0$ such that

$$
B_{\rho}(0) \subset \subset \Omega \quad \text { and } \quad \alpha_{n-1}^{-1} \rho^{-n-1} \mu_{E}\left(B_{\rho}(0)\right) \leqslant 1+\delta .
$$

Proposition 3.3 implies that $\mu_{E}$ has generalized mean curvature $\vec{H}$ in $\Omega$, given by

$$
\vec{H}=-\left(\lambda-2 \gamma \phi_{E}-f\right) v_{E}
$$

for some constant $\lambda \in \mathbb{R}$. We have

$$
\|\vec{H}\|_{L^{\infty}\left(\mu_{E}\left\llcorner B_{\rho}(0)\right)\right.} \leqslant|\lambda|+2 \gamma \sup _{B_{\rho}(0)}\left|\phi_{E}\right|+\sup _{B_{\rho}(0)}|f|=: c_{0} .
$$


With Hölder's inequality and (20) we get

$$
\left(\int_{B_{\rho}(0)}|\vec{H}|^{p} d \mu_{E}\right)^{\frac{1}{p}} \rho^{1-\frac{n-1}{p}} \leqslant c_{0}(1+\delta)^{\frac{1}{p}} \alpha_{n-1}^{\frac{1}{p}} \rho,
$$

which is less that $\delta$ provided $\rho \leqslant \delta c_{0}^{-1} 2^{-\frac{1}{p}} \alpha_{n-1}^{-\frac{1}{p}}$. Thus the hypotheses (h) are satisfied and Theorem 2.1 implies the existence of a function $u: B^{\prime}\left(:=B_{\gamma \rho}^{n-1}(0)\right) \rightarrow \mathbb{R}$ of class $C^{1, \alpha}, \alpha=1-(n-1) / p$, such that $u(0)=0, D u(0)=0$, and $\operatorname{spt}\left(\mu_{E}\right) \cap B_{\gamma \rho}(0)=\operatorname{graph}(u) \cap B_{\gamma \rho}(0)$. Moreover, our orientation assumption on $E$ implies that $\bar{E} \cap\left(B^{\prime} \times I\right)=\overline{\text { epigraph }(u)} \cap\left(B^{\prime} \times I\right)$ for some open interval $0 \in I$.

Now let $X\left(x^{\prime}, z\right)=\zeta(z) \eta\left(x^{\prime}\right) e_{n}$, where $\eta \in C_{c}^{1}\left(B^{\prime}\right), x^{\prime} \in B^{\prime}, e_{n}=(0, \ldots, 0,1)$ is the $n$-thstandard basis vector, and where $\zeta \in C_{c}^{\infty}(\mathbb{R})$ is a cut-off function such that $(\zeta \circ u)(x)=1$ for every $x \in B^{\prime}$.

Then recalling that $\operatorname{div}_{E} X=\operatorname{div} X-v_{E} \cdot D X v_{E}$, we have $\operatorname{div}_{E} X=-\left(\nabla^{\prime} \eta, 0\right) \cdot v_{E} v_{E}^{n}$ where $v_{E}^{n}$ is the $n$-th component of the normal vector, and where $\nabla^{\prime}$ is the gradient in $\mathbb{R}^{n-1}$. Since $\partial^{*} E \cap$ $\left(B^{\prime} \times I\right)=\partial E \cap\left(B^{\prime} \times I\right)$ is the graph of $u$, and by our orientation assumption, we have that $v_{E}^{n}=\frac{-1}{\sqrt{1+\left|\nabla^{\prime} u\right|^{2}}}$. Using the area formula, equation (19) becomes

$$
-\int_{B^{\prime}} \frac{\nabla^{\prime} \eta \cdot \nabla^{\prime} u}{\sqrt{1+\left|\nabla^{\prime} u\right|^{2}}} d x^{\prime}=\int_{B^{\prime}}\left(\lambda-2 \gamma v_{E}\left(x^{\prime}, u\right)-f\left(x^{\prime}, u\right)\right) \eta d x^{\prime} .
$$

Equation (21) is the weak form of the prescribed mean curvature equation. Since by Theorem 2.1 the gradient of $u$ is locally uniformly bounded in $C^{0, \alpha}$ and since the right hand side of (21) is of class $C^{1, \alpha}$, interior Schauder estimates (see [10]) and bootstrapping imply local $C^{3, \alpha}$ regularity of the function $u$. Thus (21) holds pointwise, and since $x_{0} \in \partial_{\Omega}^{*} E$ was arbitrary we have

$$
H+2 \gamma \phi_{E}+f=\lambda \text { on } \partial_{\Omega}^{*} E,
$$

where $H$ is the classical mean curvature of the surface $\partial_{\Omega}^{*} E$.

\subsection{On the size of the singular set}

By a direct consequence of the monotonicity formula, see [26, Corollary 17.8], we have that $\Theta\left(\mu_{E}, x\right)$ exists and that $\Theta\left(\mu_{E}, x\right) \geqslant 1$ for every point $x \in \operatorname{spt}\left(\mu_{E}\right)=\overline{\partial E \cap \Omega}$. This allows us to estimate the size of the singular set $\left(\partial E \backslash \partial^{*} E\right) \cap \Omega$.

Proposition 4.1 We have the following estimate

$$
\mathcal{H}^{n-1}\left(\left(\partial E \backslash \partial^{*} E\right) \cap \Omega\right)=0 .
$$

Proof. The proof is exactly the same as the proof of [21, Theorem 2 of Section 2.5.4], but now using the monotonicity formula [26, Theorem 17.7] to obtain the required lower bound on area. More precisely, the monotonicity formula [26, Theorem 17.7] implies that

$$
\mu_{E}\left(B_{\rho_{j}}\left(x_{j}\right)\right) \geqslant \frac{\alpha_{n-1}}{2} \rho_{j}^{n-1},
$$


if $\delta$ is small enough as to guarantee that

$$
\alpha_{n-1}^{\frac{n-1}{p}}\left(1-2^{-\frac{1}{p}}\right) \geqslant \frac{\|\vec{H}\|_{L^{p}}}{p-(n-1)} \delta^{1-\frac{n-1}{p}},
$$

for some $p>n-1$.

\section{Boundary regularity of local minimizers}

In this section we outline how Theorem 1.2 can be used to prove boundary regularity, that is regularity near points $x \in \partial \Omega \cap \partial E$, for local minimizers $E$ of $\varepsilon_{\gamma}$ in $Q$ or $Q_{m}$. This has already been established in [17] but we include it for convenience of the reader.

As mentioned earlier, the interior regularity for local minimizers of $\varepsilon_{\gamma}$ was proved by Sternberg and Topaloglu [27, Propostion 2.1]. The authors prove that local minimizers of $\varepsilon_{\gamma}$ are $(K, \varepsilon)$ minimal and can thus appeal to the standard methods (cf. [20]). We include a slightly different proof.

DEFINITION 5.1 We say that $E \in Q$ or $Q_{m}$ is a local minimizer of $\varepsilon_{\gamma}$ in $Q$ or $Q_{m}$ (at scale $R$ ) if for all balls $B_{R}(x) \subset \mathbb{R}^{n}$ we have that

$$
\varepsilon_{\gamma}(E) \leqslant \varepsilon_{\gamma}(F) \text { for all } F \in Q \text { or } Q_{m} \text { with } E \Delta F \subset \subset B_{R}(x) .
$$

REMARK 5.2 Theorem 1.2 implies that for any ball $B_{\rho}(x) \subset \mathbb{R}^{n}$ with $0<\left|E \cap B_{\rho}(x)\right|<\mid \Omega \cap$ $B_{\rho}(x) \mid$ we can find exterior and interior points, i.e. there exist two balls $B_{r}(a), B_{r}(b) \subset \subset \Omega \cap$ $B_{\rho}(x)$ with $\bigcup_{t \in[0,1]} B_{r}(t a+(1-t) b) \subset \subset \Omega$ such that

$$
\left|B_{r}(a) \backslash E\right|=\left|E \cap B_{r}(a)\right|=0 .
$$

We are now ready to prove the following.

Proposition 5.3 (cf. [27, Propostion 2.1] ) Let $E \in Q$ or $Q_{m}$ be a local minimizer of $\varepsilon_{\gamma}$ in $Q$ or $Q_{m}$ at scale $2 R_{0}>0$, and let $0<\left|E \cap B_{R_{0}}\left(x_{0}\right)\right|<\left|\Omega \cap B_{R_{0}}\left(x_{0}\right)\right|$ for some ball $B_{R_{0}}\left(x_{0}\right) \subset \mathbb{R}^{n}$. Then $E$ is $(K, \varepsilon)$-minimal in $B_{R}\left(x_{0}\right)$ for some $R \leqslant R_{0}$, that is for every $B_{\rho} \subset \subset B_{R}\left(x_{0}\right)$

$$
P(E, \Omega) \leqslant P(F, \Omega)+K \rho^{n} \quad \text { for all } F \text { such that } F \Delta E \subset \subset B_{\rho} .
$$

Proof. Let $B_{\rho} \subset \subset B_{R}\left(x_{0}\right)$ and let $F$ be such that $F \Delta E \subset \subset B_{\rho}$. We only give a proof for local minimizers in $Q$. (For the case with a volume constraint one may use Remark 5.2 to adjust the volume of the competitor $F$ which gives us the additional term $\frac{c(n)}{r} \rho^{n}$ on the right hand side of equation (23) below. We refer to [15, Proposition 1] for details. Alternatively, one can proceed as in [27] and use a result of Giusti [12, Lemma 2.1] to balance out the volume constraint.)

By (22) we have that

$$
\begin{aligned}
P(E, \Omega) \leqslant P(F, \Omega)+\gamma \int_{F} \int_{F} G(x, y) d x d y-\gamma \int_{E} \int_{E} G(x, y) d x d y & \\
& \quad+\int_{F} f d x-\int_{E} f d x .
\end{aligned}
$$


The last two terms can be estimated by $\int_{\Omega \cap B_{\rho}} f d x \leqslant c(n)\|f\|_{L \infty} \rho^{n}$, cf. [20]. In remains to estimate the difference of the nonlocal terms. Setting $A:=\Omega \cap B_{R}\left(x_{0}\right)$, we estimate for any $p>n$

$$
\begin{aligned}
& \int_{F} \int_{F} G(x, y) d x d y-\int_{E} \int_{E} G(x, y) d x d y \\
& \quad \leqslant \int_{F}\left(\int_{F \cap B_{\rho}} G(x, y) d x-\int_{E \cap B_{\rho}} G(x, y) d x\right) d y+\int_{E \Delta F}\left|\phi_{E}\right| d x \\
& \quad \leqslant \int_{E \cup\left(\Omega \cap B_{\rho}\right)}\left(\int_{\Omega \cap B_{\rho}}\left|\chi_{F \cap B_{\rho}}(x)-\chi_{E \cap B_{\rho}}(x)\right||G(x, y)| d x\right) d y+\int_{B_{\rho}}\left|\phi_{E}\right| d x \\
& \quad \leqslant\|G\|_{L^{1}((E \cup A) \times A)}\left|B_{\rho}\right|+\left(\int_{A}\left|\phi_{E}\right|^{p} d x\right)^{\frac{1}{p}}\left|B_{\rho}\right|^{1-\frac{1}{p}} \\
& \quad \leqslant c(n, p, G, E) \rho^{n-1+\left(1-\frac{n}{p}\right)} .
\end{aligned}
$$

The claim follows with $\varepsilon=1-\frac{n}{p}$ for any $p>n$ and $K=c(n, p, G, E)$ (or $K=c(n, p, G, E, r)$ in case of a volume constraint with $r$ as in Remark 5.2).

Definition 5.4 (cf. [16]) Here $\operatorname{reg}\left(\mu_{E}\right)$ is defined as the set of all points in $\partial E \cap \bar{\Omega}=\operatorname{spt}\left(\mu_{E}\right)$ such that one of the following alternatives holds.

1. If $x \in \operatorname{reg}\left(\mu_{E}\right) \cap \Omega$ there exits an oriented $C^{1}$-hypersurface $M_{x}$ such that $\mu_{E}=\mathcal{H}^{n-1}\left\llcorner M_{x}\right.$ and $v_{E}=v_{M_{x}}$ in a neighborhood of $x$.

2. If $x \in \operatorname{reg}\left(\mu_{E}\right) \cap \partial \Omega$ there exits an oriented $C^{1}$-hypersurface $M_{x}^{\prime}$ with boundary inside $\partial \Omega$ such that $\mu_{E}=H^{n-1}\left\llcorner M_{x}^{\prime}\right.$ and $v_{E}=v_{M_{x}^{\prime}}$ in a neighborhood of $x$.

And $\operatorname{sing}\left(\mu_{E}\right):=\overline{\partial E \cap \Omega} \backslash \operatorname{reg}\left(\mu_{E}\right)$.

Theorem 1.2 and Proposition 5.3 in conjunction with the results of Grüter [16] in which boundary regularity of $(K, \varepsilon)$-minimizers with weakly orthogonal surface measure was shown, immediately imply the following

THEOREM 5.5 (cf. [17, Theorem 3.2]) Let $E \in Q$ or $Q_{m}$ be a local minimizer of $\varepsilon_{\gamma}$ in $Q$ or $Q_{m}$. Then

1. $\operatorname{reg}\left(\mu_{E}\right)$ is of class $C^{1, \alpha}$ for all $\alpha \in(0,1), \operatorname{reg}\left(\mu_{E}\right) \cap \Omega$ is of class $C^{3, \alpha}$ for all $\alpha \in(0,1)$ and has mean curvature $H=\lambda-2 \gamma \phi_{E}-f$ for some constant $\lambda \in \mathbb{R}$. If $x \in \operatorname{reg}\left(\mu_{E}\right) \cap \partial \Omega$ then $\operatorname{reg}\left(\mu_{E}\right)$ and $\partial \Omega$ intersect orthogonally in a neighborhood of $x$.

2. $\mathcal{H}^{s}\left(\operatorname{sing}\left(\mu_{E}\right)\right)=0$ for all $s>n-8$.

REMARK 5.6 In case $\Omega$ is of class $C^{k, \alpha}$ for $k=2,3$ we get that $\operatorname{reg}\left(\mu_{E}\right)$ is of class $C^{k, \alpha}$ (up to the boundary).

Acknowledgments. The research of the first-named author was supported by the Herchel Smith Research Fellowship at the University of Cambridge and NSF grant DMS-0807347. The firstnamed author would like to thank Theodora Bourni and Robert Haslhofer for helpful discussions throughout the course of this work. 


\section{REFERENCES}

1. Allard, W. K., On the first variation of a varifold. Ann. of Math. (2) 95 (1972), 417-491. Zblo252. 49028 MR0307015

2. Chen, G.-Q., Torres, M., \& Ziemer, W. P., Gauss-Green theorem for weakly differentiable vector fields, sets of finite perimeter, and balance laws. Comm. Pure Appl. Math. 62 (2009), 242-304. Zbl1158. 35062 MR2468610

3. Chen, L. Q., \& Khachaturyan, A. G., Dynamics of simultaneous ordering and phase separation and effect of long-range coulomb interactions. Phys. Rev. Lett. 70 (Mar 1993), 1477-1480.

4. Choksi, R., \& Sternberg, P., On the first and second variations of a nonlocal isoperimetric problem. J. Reine Angew. Math. 611 (2007), 75-108. Zbl1132. 35029 MR2360604

5. Cicalese, M., \& Spadaro, E., Droplet minimizers of an isoperimetric problem with long-range interactions. Comm. Pure Appl. Math. 66 (2013), 1298-1333. Zbl1269. 49085 MR3069960

6. De Gennes, P.-G., Effect of cross-links on a mixture of polymers. Journal de Physique Lettres 40 (1979), 69-72.

7. Emery, V., \& Kivelson, S., Frustrated electronic phase separation and high-temperature superconductors. Physica C: Superconductivity 2094 (1993), 597 - 621.

8. EVAns, L. C., \& GARIEPY, R. F., Measure theory and fine properties of functions. Studies in Advanced Mathematics. CRC Press, Boca Raton, FL, 1992. Zbl0804.28001 MR1158660

9. Giaquinta, M., \& Hildebrandt, S., Calculus of variations. I, vol. 310 of Grundlehren der Mathematischen Wissenschaften [Fundamental Principles of Mathematical Sciences]. Springer-Verlag, Berlin, 1996. The Lagrangian formalism. Zb10853.49001 MR1368401

10. Gilbarg, D., \& Trudinger, N. S., Elliptic partial differential equations of second order. Classics in Mathematics. Springer-Verlag, Berlin, 2001. Reprint of the 1998 edition. Zbl1042. 35002 MR1814364

11. GiUst, E., Minimal surfaces and functions of bounded variation, vol. 80 of Monographs in Mathematics. Birkhäuser Verlag, Basel. Zb10545.49018 MR0775682

12. Giusti, E., The equilibrium configuration of liquid drops. J. Reine Angew. Math. 321 (1981), 53-63. Zb10438.76078 MR0597979

13. Glotzer, S. C., Di Marzio, E. A., \& Muthukumar, M., Reaction-controlled morphology of phaseseparating mixtures. Phys. Rev. Lett. 74 (Mar 1995), 2034-2037.

14. Goldman, D., Asymptotics of non-minimizing stationary points of the ohta-kawasaki energy. arXiv arXiv:1410.7047 [math.AP] (2014).

15. Gonzalez, E., Massari, U., \& TAMAnini, I., On the regularity of boundaries of sets minimizing perimeter with a volume constraint. Indiana Univ. Math. J. 32 (1983), 25-37. Zbl0486.49024 MR0684753

16. GRÜTER, M., Boundary regularity for solutions of a partitioning problem. Arch. Rational Mech. Anal. 97 (1987), 261-270. Zbl0613.49029 MR0862549

17. Julin, V., \& Pisante, G., Minimality via second variation for microphase separation of diblock copolymer melts. J. Reine Angew. Math. (2015).

18. Lundqvist, S., \& March, N. H., Theory of the Inhomogeneous Electron Gas. Physics of Solids and Liquids. Springer, 1983.

19. Maggi, F., Sets of finite perimeter and geometric variational problems, vol. 135 of Cambridge Studies in Advanced Mathematics. Cambridge University Press, Cambridge, 2012. An introduction to geometric measure theory. Zbl1255.49074 MR2976521

20. Massari, U., Esistenza e regolarità delle ipersuperfice di curvatura media assegnata in $R^{n}$. Arch. Rational Mech. Anal. 55 (1974), 357-382. Zbl0305. 49047 MR0355766

21. Massari, U., \& Miranda, M., Minimal surfaces of codimension one, vol. 91 of North-Holland Mathematics Studies. North-Holland Publishing Co., Amsterdam, 1984. Notas de Matemática [Mathematical Notes], 95. Zbl0565.49030 MR0795963 
22. NAgAev, E. L. Phase separation in high-temperature superconductors and related magnetic systems. Physics-Uspekhi 38 (1995), 497.

23. OHTA, T., \& KAWASAKI, K., Equilibrium morphologies of block copolymer melts. Macromolecules 19 (1986), 2621-2632.

24. REN, X., \& WEI, J., On the multiplicity of solutions of two nonlocal variational problems. SIAM J. Math. Anal. 31 (2000), 909-924 (electronic). Zbl0973.49007 MR1752422

25. RÖGER, M., \& Tonegawa, Y. Convergence of phase-field approximations to the Gibbs-Thomson law. Calc. Var. Partial Differential Equations 32 (2008), 111-136. Zbl1159. 49046 MR2377408

26. Simon, L. Lectures on geometric measure theory, vol. 3 of Proceedings of the Centre for Mathematical Analysis, Australian National University. Australian National University, Centre for Mathematical Analysis, Canberra, 1983. Zbl0546.49019 MR0756417

27. Sternberg, P., \& Topaloglu, I. On the global minimizers of a nonlocal isoperimetric problem in two dimensions. Interfaces Free Bound. 13 (2011), 155-169. Zbl1216. 35019 MR2793856

28. Volkmann, A., Regularity of isoperimetric hypersurfaces with obstacles in Riemannian manifolds. Diplomarbeit, Albert-Ludwigs-Universität Freiburg (2010). 